\title{
A quantum stochastic approach to the spectrum of a two-level atom
}

\author{
Alberto Barchiellitif and Nicola Pero \\ Dipartimento di Matematica, Politecnico di Milano, \\ Piazza Leonardo da Vinci 32, I-20133 Milano, Italy
}

\begin{abstract}
By means of quantum stochastic calculus we construct a model for an atom with two degenerate levels and stimulated by a laser and we compute its fluorescence spectrum; let us stress that, once the model for the unitary atom-field dynamics has been given, then the spectrum is computed without further approximations. If only the absorption/emission term is included in the interaction, we reobtain the Mollow spectrum in the case of a monochromatic laser and the Kimble-Mandel spectrum in the case of a "phase diffusion model" for a non monochromatic laser. However, our model can describe also another type of light scattering, a "direct scattering" due to the response of the atom as a whole, which we expect to be small, but which interferes with the scattering due to the absorption/emission channel. When both the scattering channels are introduced we obtain a modification of the Mollow-Kimble-Mandel spectrum, which shares the main features with the usual case, but which presents some asymmetries even in the case of no detuning.

PACS numbers: 42.50.-p, 42.50.Ct, 42.50.Hz, 02.50.Ey
\end{abstract}

\section{Introduction}

Quantum stochastic calculus (QSC) [1]- [4] is a noncommutative analogue of the classical Ito's calculus and it has had many applications in modelling quantum optical systems [5]-[14]. In particular, inside QSC a Schrödinger equation for unitary operators can be formulated, the Hudson-Parthasarathy equation (5) [1], which is well suited to treat the interaction between matter and electromagnetic field within the usual approximation schemes of quantum optics [12].

The simplest, non trivial matter-field system is a two-level atom stimulated by a laser. The fluorescence spectrum of such a system, in the case of a perfectly monochromatic laser, was obtained by Mollow [15]; then, the case of a laser with a Lorentzian spectrum was developed by Kimble and Mandel [16, 17. QSC and Hudson-Parthasarathy equation give not only the way of giving an unified treatment of such a system and of connecting it to the photon-detection theory, but they allow also to explore possible corrections to the dynamics and to obtain modifications of the Mollow spectrum. In the usual treatments, the scattering of the light by the atom is described by the absorption/emission process due to the electric dipole interaction involving only two states of the atom. But even if the absorption/emission process would be forbidden and the atom would be frozen in the up or down level, some $\ddagger$ Also: Istituto Nazionale di Fisica Nucleare, Sezione di Milano. 
scattering of light would remain, due to the response of the atom as a whole; we can call it "direct scattering". For instance, in a perturbative development in Feynman graphs, scattering processes would be generated also by virtual transitions starting and ending in one of the two states left in the description, but involving as intermediate states the other ones, which have been eliminated in the final description. The use of Hudson-Parthasarathy equation allows, even when the atom is approximated by a two-level system, for the introduction of both the "absorption/emission channel" and the "direct scattering channel". This modified model, in the case of a perfectly monochromatic laser, has been presented in [18] and developed in [19]; the first results for a non monochromatic laser have been obtained in [20].

In Section 2 we give the mathematical model for the dynamics of the two-level atom interacting with the electromagnetic field, including the polarization of the light and the degeneracy of the up and down levels, which has never been considered before and which has an effect on the angular dependence of the spectrum. We consider the usual dipole interaction and the possible corrections giving the direct scattering; the so called phase diffusion model is used for the laser as in 16]. In Section 3 the general formulae for the spectrum are obtained and its angular dependence is discussed. Then, the total spectrum, integrated over the whole solid angle, is studied. In the case of only the absorption/emission process in the interaction term, we reobtain the Mollow spectrum 15 for a perfectly monochromatic laser and the Kimble-Mandel spectrum [16] for a non monochromatic laser with Lorentzian spectrum. In the case of the modified model, when also the direct scattering is included, but the laser is perfectly monochromatic, we reobtain the results of [19], although there the level degeneracy was not taken into account. According to the values of the various parameters, the fluorescence spectrum can present a well resolved triplet structure as in the Mollow spectrum, but also it can be distorted and made asymmetric by the presence of the new terms. Experiments [21]-25] confirm essentially the triplet structure; some asymmetry has been found and its origin has been attributed to various causes. The presence of the new terms we have introduced could give a model for treating such an asymmetry. When the laser bandwidth is taken into account, the modified model gives corrections to the spectrum found in 16] by Kimble and Mandel.

\section{The model}

\subsection{The unitary atom-field dynamics}

2.1.1. Fock space and the Hudson-Parthasarathy equation. Let us recall the rules of QSC and the Hudson-Parthasarathy equation; this is just to fix our notations, while for the proper mathematical presentation we refer to the book by Parthasarathy [4].

We denote by $\mathcal{F}$ the Boson Fock space over the "one-particle space" $\mathcal{Z} \otimes L^{2}\left(\mathbb{R}_{+}\right)$, where $\mathcal{Z}$ is a separable complex Hilbert space; we shall see in section 2.1 .3 how to choose $\mathcal{Z}$. A vector $f$ in $\mathcal{Z} \otimes L^{2}\left(\mathbb{R}_{+}\right) \simeq L^{2}\left(\mathbb{R}_{+} ; \mathcal{Z}\right)$ is a square integrable function from $\mathbb{R}_{+}$into $\mathcal{Z}$. Let $\left\{h_{k}, k \geq 1\right\}$ be a c.o.n.s. in $\mathcal{Z}$ and let us denote by $A_{k}(t)$, $A_{k}^{\dagger}(t), \Lambda_{k l}(t)$ the annihilation, creation and gauge (or number) processes associated with such a c.o.n.s.; we can write formally $A_{k}(t)=\int_{0}^{t} a_{k}(s) \mathrm{d} s, A_{k}^{\dagger}(t)=\int_{0}^{t} a_{k}^{\dagger}(s) \mathrm{d} s$, $\Lambda_{k l}(t)=\int_{0}^{t} a_{k}^{\dagger}(s) a_{l}(s) \mathrm{d} s$, where $a_{k}(t), a_{k}^{\dagger}(t)$ are usual Bose fields, satisfying the canonical commutation rules $\left[a_{k}(t), a_{l}^{\dagger}(s)\right]=\delta_{k l} \delta(t-s), \ldots$. The Fock space $\mathcal{F}$ is spanned by the coherent vectors $e(f), f \in L^{2}\left(\mathbb{R}_{+} ; \mathcal{Z}\right)$, whose components in the 
$0,1, \ldots, n, \ldots$ particle spaces are

$$
e(f)=\exp \left(-\frac{1}{2}\|f\|^{2}\right)\left(1, f,(2 !)^{-1 / 2} f \otimes f, \ldots,(n !)^{-1 / 2} f^{\otimes n}, \ldots\right) ;
$$

in particular the vector $e(0)$ is the Fock vacuum. The coherent vectors (1) are normalized and they are the eigenstates of the annihilation operators; indeed, we have $A_{k}(t) e(f)=\int_{0}^{t}\left\langle h_{k} \mid f(s)\right\rangle \mathrm{d} s e(f)$. In QSC integrals of "Ito type" with respect to $\mathrm{d} A_{k}(t), \mathrm{d} A_{k}^{\dagger}(t), \mathrm{d} \Lambda_{k l}(t)$ are defined. The main practical rules to manipulate "Ito differentials" are the facts that $\mathrm{d} A_{k}(t), \mathrm{d} A_{k}^{\dagger}(t), \mathrm{d} \Lambda_{k l}(t)$ commute with anything containing the fields only up to time $t$ (objects called adapted processes) and that the products of the fundamental differentials satisfy $\mathrm{d} A_{k}(t) \mathrm{d} A_{l}^{\dagger}(t)=$ $\delta_{k l} \mathrm{~d} t, \mathrm{~d} A_{k}(t) \mathrm{d} \Lambda_{r l}(t)=\delta_{k r} \mathrm{~d} A_{l}(t), \mathrm{d} \Lambda_{k r}(t) \mathrm{d} A_{l}^{\dagger}(t)=\delta_{r l} \mathrm{~d} A_{k}^{\dagger}(t), \mathrm{d} \Lambda_{k r}(t) \mathrm{d} \Lambda_{s l}(t)=$ $\delta_{r s} \mathrm{~d} \Lambda_{k l}(t)$; all the other products and the products involving $\mathrm{d} t$ vanish.

Let $\mathcal{H}$ be a separable complex Hilbert space (the system space) and let $R_{k}, k \geq 1$, $S_{k l}, k, l \geq 1, H$ be bounded operators in $\mathcal{H}$ such that $H^{\dagger}=H, \sum_{k} R_{k}^{\dagger} R_{k}$ is strongly convergent to a bounded operator, and

$$
\sum_{k l} S_{k l} \otimes\left|h_{k}\right\rangle\left\langle h_{l}\right|=: S \in \mathcal{U}(\mathcal{H} \otimes \mathcal{Z}),
$$

$\mathcal{U}(*)$ "means unitary operators on $*$ "; we set also

$$
\begin{aligned}
& R: \mathcal{H} \rightarrow \mathcal{H} \otimes \mathcal{Z}, \quad R \xi:=\sum_{k}\left(R_{k} \xi\right) \otimes h_{k}, \\
& K=H-\frac{\mathrm{i}}{2} \sum_{k} R_{k}^{\dagger} R_{k} \equiv H-\frac{\mathrm{i}}{2} R^{\dagger} R .
\end{aligned}
$$

Then (see reference [4] Theorem 27.8 p. 228) there exists a unique unitary operatorvalued adapted process $U(t)$ satisfying $U(0)=11$ and

$\mathrm{d} U(t)=\left\{\sum_{k} R_{k} \mathrm{~d} A_{k}^{\dagger}(t)+\sum_{k l}\left(S_{k l}-\delta_{k l}\right) \mathrm{d} \Lambda_{k l}(t)-\sum_{k l} R_{k}^{\dagger} S_{k l} \mathrm{~d} A_{l}(t)-\mathrm{i} K \mathrm{~d} t\right\} U(t) .(5)$

The Bose fields introduced here represent a good approximation of the electromagnetic field in the so called quasi-monochromatic paraxial approximation [26, 12]. Now, $\mathcal{F}$ is interpreted as the Hilbert space of the electromagnetic field; $A_{k}^{\dagger}(t)$ creates a photon with state $h_{k}$ in the time interval $[0, t], A_{k}(t)$ annihilates it, $\Lambda_{k k}(t)$ is the selfadjoint operator representing the number of photons with state $h_{k}$ in the time interval $[0, t]$. The operator $U(t)$ represents the evolution operator for a compound system (say an atom plus the electromagnetic field), in the interaction picture with respect to the free dynamics of the field.

2.1.2. The two-level atom. The experiments 21 25] involve or the hyperfine component of the $D_{2}$ line of sodium with up level $2 P_{3 / 2}, F=3$ and down level $2 S_{1 / 2}, F=2$, or the levels $6 s 6 p{ }^{1} P_{1}, F=1$, and $6 s^{2} S_{0}, F=0$ of ${ }^{138} \mathrm{Ba} ; F$ is the total angular momentum.

In order to describe an atom with two degenerate levels as in the experimental situation, we take

$$
\mathcal{H}=\mathbb{C}^{2 F_{-}+1} \oplus \mathbb{C}^{2 F_{+}+1}, \quad F_{+}=F_{-}+1,
$$

where $F_{-}$is integer or semi-integer. We denote by $\left|F_{ \pm}, M\right\rangle, M=-F_{ \pm}, \ldots, F_{ \pm}$, the angular momentum basis in $\mathcal{H}$; the parities of the states of the two levels 
must be opposed, let us say they are $\epsilon_{ \pm}$, with $\epsilon_{+} \epsilon_{-}=-1$. Let us denote by $P_{ \pm}=\sum_{M=-F_{ \pm}}^{F_{ \pm}}\left|F_{ \pm}, M\right\rangle\left\langle F_{ \pm}, M\right|$ the two projection on the up or down states. Then the free energy $H$, contained in the quantity (画), must be given by

$$
H=\frac{1}{2} \omega_{0}\left(P_{+}-P_{-}\right), \quad \omega_{0}>0,
$$

where the atomic frequency $\omega_{0}$ must already include the Lamb shift.

2.1.3. The photon space. In the approximations we are considering [26, 12], the fields behave as monodimensional waves, so that a change of position is equivalent to a change of time and viceversa. Then, the space $\mathcal{Z}$ has to contain only the degrees of freedom linked to the direction of propagation and to the polarization. To describe a spin-1 0-mass particle we use the conventions of Messiah [27] pp. 550, 1032-1037.

A c.o.n.s. in $\mathcal{Z}$ is given by $|j, m ; \varpi\rangle \equiv \vec{\Theta}_{j m}^{\varpi}, j=1,2, \ldots, m=-j,-j+1, \ldots, j$, $\varpi= \pm 1 ; j m$ is the total angular momentum, $\varpi=+1$ denotes electrical multipole, $\varpi=-1$ denotes magnetic multipole and $(-1)^{j} \varpi$ is the parity. By using the spherical harmonics $Y_{l m}(\theta, \phi)$ and the orbital angular momentum operator $\vec{\ell}$, one has

$$
\vec{\Theta}_{j m}^{-1}=\frac{1}{\sqrt{j(j+1)}} \vec{\ell} Y_{j m}, \quad \vec{\Theta}_{j m}^{+1}=\mathrm{i} \vec{p} \times \vec{\Theta}_{j m}^{-1},
$$

where $\vec{p}$ is the direction versor given by $p_{1}=\sin \theta \cos \phi, p_{2}=\sin \theta \sin \phi, p_{3}=\cos \theta$.

2.1.4. The interaction. Let us consider now the terms with the creation and annihilation operators in the dynamical equation (5); they must describe the absorption/emission process. By asking spherical symmetry, parity conservation and only electrical dipole contribution in $R$, we must have

$$
\begin{aligned}
R & =\alpha \sum_{M=-F_{+}}^{F_{+}}\left|\epsilon_{+} ; F_{-} ; 1 ; F_{+}, M\right\rangle\left\langle F_{+}, M\right|, \quad \alpha \in \mathbb{C}, \quad \alpha \neq 0, \\
\left|\epsilon_{+} ; F_{-} ; 1 ; F_{+}, M\right\rangle & =\sum_{m_{1}=-F_{-}}^{F_{-}} \sum_{m_{2}=-1}^{1}\left|F_{-}, m_{1}\right\rangle \otimes\left|1, m_{2} ;+1\right\rangle\left\langle F_{-}, m_{1} ; 1, m_{2} \mid F_{+}, M\right\rangle,
\end{aligned}
$$

where by $\left\langle F_{-}, m_{1} ; 1, m_{2} \mid F_{+}, M\right\rangle$ we denote the Clebsch-Gordan coefficients (27] pp. 560-563). The interaction terms containing $R$ are responsible for the spontaneous decay of the atom and, as we shall see, $|\alpha|^{2}$ turns out to be the natural line width. Note that

$$
R_{k}=P_{-} R_{k} P_{+}, \quad R^{\dagger} R=|\alpha|^{2} P_{+} .
$$

The interaction term containing the $\Lambda$-process must give the residual scattering when the atom is frozen in the up or down level, so we take the unitary operator $S$ of the form

$$
S=P_{+} \otimes S^{+}+P_{-} \otimes S^{-}, \quad S^{ \pm} \in \mathcal{U}(\mathcal{Z}) .
$$

Then, by spherical symmetry and parity conservation we must have

$$
S^{ \pm}=\sum_{j=1}^{\infty} \sum_{m=-j}^{j} \sum_{\varpi= \pm 1}|j, m ; \varpi\rangle \exp \left\{2 \mathrm{i} \delta_{ \pm}(j ; \varpi)\right\}\langle j, m ; \varpi|,
$$

$0 \leq \delta_{ \pm}(j ; \varpi)<2 \pi$; the $\delta$ 's are the phase shifts for the atom frozen in the up or down level. Quantities like $\omega_{0}, \alpha, \delta_{ \pm}(j ; \varpi)$ are phenomenological parameters, or, better, they have to be computed from some more fundamental theory, such as some approximation to quantum electrodynamics. 


\subsection{Some dynamical features of the model}

2.2.1. The master equation. Equation (5) already contains the usual Markov approximations; indeed, when the initial state of the field is a coherent vector, then the atomic reduced dynamics turns out to be given by a master equation without need of introducing further approximations [1, 1, 12]. If $\rho_{0}$ is the initial atomic density matrix on $\mathcal{H}$ and the coherent vector $e(f)$ is the state of the field, then the atomic reduced statistical operator

$$
\rho(t ; f)=\operatorname{Tr}_{\mathcal{F}}\left\{U(t)\left(\rho_{0} \otimes|e(f)\rangle\langle e(f)|\right) U(t)^{\dagger}\right\}
$$

turns out to satisfy the master equation

$$
\frac{\mathrm{d}}{\mathrm{d} t} \rho(t ; f)=\mathcal{L}(f(t))[\rho(t ; f)]
$$

where the time dependent Liouvillian turns out to be

$$
\begin{aligned}
\mathcal{L}(f(t))[\rho]=-\mathrm{i}[ & H(f(t)), \rho] \\
+ & \frac{1}{2} \sum_{k}\left(\left[R_{k}(f(t)) \rho, R_{k}(f(t))^{\dagger}\right]+\left[R_{k}(f(t)), \rho R_{k}(f(t))^{\dagger}\right]\right), \\
& R_{k}(f(t))=R_{k}+\left\langle h_{k} \mid S^{+} f(t)\right\rangle P_{+}+\left\langle h_{k} \mid S^{-} f(t)\right\rangle P_{-}, \\
& H(f(t))=H+\frac{\mathrm{i}}{2} \sum_{k}\left(\left\langle S^{-} f(t) \mid h_{k}\right\rangle R_{k}-\left\langle h_{k} \mid S^{-} f(t)\right\rangle R_{k}^{\dagger}\right) .
\end{aligned}
$$

Note that, when the field is in the Fock vacuum, only spontaneous emission is present; indeed, for $f(t)=0$, the Liouvillian (16a) reduces to

$$
\begin{aligned}
& \mathcal{L}(0)[\rho]=-\frac{\mathrm{i}}{2} \omega_{0}\left[P_{+}-P_{-}, \rho\right]-\frac{1}{2}|\alpha|^{2}\left(P_{+} \rho+\rho P_{+}\right)+|\alpha|^{2} \sum_{m=-1}^{1} A_{m} \rho A_{m}^{\dagger}, \\
& A_{m}=\sum_{m_{1}=-F_{-}}^{F_{-}}\left|F_{-}, m_{1}\right\rangle\left\langle F_{-}, m_{1} ; 1, m \mid F_{+}, m_{1}+m\right\rangle\left\langle F_{+}, m_{1}+m\right|,
\end{aligned}
$$

which describes decay according to the usual selection rules for electric dipole and fixes the meaning of $|\alpha|^{2}$.

2.2.2. The balance equation for the number of photons. Let us introduce the observables "total number of photons in the time interval $[0, t]$ " before and after the interaction with the atom

$$
N^{\text {in }}(t)=\sum_{k} \Lambda_{k k}(t), \quad N^{\text {out }}(t)=U^{\dagger}(t) N^{\text {in }}(t) U(t) .
$$

By using the computations on the increments of the "output fields" given in [12], we obtain in the present model the balance equation

$$
N^{\text {in }}(t)+\frac{1}{2}\left(P_{+}-P_{-}\right)=N^{\text {out }}(t)+\frac{1}{2} U(t)^{\dagger}\left(P_{+}-P_{-}\right) U(t) .
$$

Such an equation says that the number of photons entering the system up to time $t$ plus the photons stored in the atom at time 0 is equal to the number of photons leaving the system up to time $t$ plus the photons stored in the atom at time $t$. An equation for mean values similar to (19) has been used for restricting the possible forms of the interaction term in the simpler model studied in [19]. 


\subsection{The laser and the equilibrium state}

2.3.1. The phase diffusion model for the laser. As in [16, 17] we take as the state of the laser a mixture of coherent vectors $\mathbb{E}[|e(f)\rangle\langle e(f)|]$ with

$f(t)=\exp \{-\mathrm{i}(\omega t+\sqrt{B} W(t))\} \theta(T-t) \lambda, \quad \lambda \in \mathcal{Z}, \quad \omega>0, \quad B \geq 0$,

$W(t)$ is a standard Wiener process, $\theta$ is the usual step function and $T$ is a large time; $T \rightarrow+\infty$ in the final results is always understood and $\mathbb{E}$ means the classical expectation over the Wiener process. Note that this model of the laser implies a Lorentz spectrum of bandwidth $B$; indeed, for $T \rightarrow \infty$, one obtains

$$
\frac{\hbar \omega}{2 \pi} \int_{-\infty}^{+\infty} \mathrm{e}^{\mathrm{i} \nu \tau} \mathbb{E}[\langle f(t) \mid f(t+\tau)\rangle] \mathrm{d} \tau=\hbar \omega\|\lambda\|^{2} \frac{B /(2 \pi)}{(\nu-\omega)^{2}+B^{2} / 4} .
$$

The whole model is meaningful only for $\omega$ not too "far" from $\omega_{0}$.

In order to describe a well collimated laser beam propagating along the direction $z(\theta=0)$ and with right circular polarization, we take

$$
\begin{aligned}
& \lambda=\alpha \Omega \mathrm{e}^{\mathrm{i} \delta} \lambda_{+}, \quad \Omega>0, \quad \delta \in[0,2 \pi) \\
& \vec{\lambda}_{+}(\theta, \phi)=\frac{1_{[0, \Delta \theta]}(\theta)}{\Delta \theta \sqrt{3 \pi(1-\cos \Delta \theta)}}\left(-\frac{1}{\sqrt{2}}\right)(\vec{i}+\mathrm{i} \vec{j}),
\end{aligned}
$$

where $1_{[0, \Delta \theta]}(\theta)=1$ for $0 \leq \theta \leq \Delta \theta, 1_{[0, \Delta \theta]}(\theta)=0$ elsewhere; in all the physical quantities the limit $\Delta \theta \downarrow 0$ will be taken. Note that the power of the laser $\hbar \omega\|\lambda\|^{2}=\frac{2}{3} \hbar \omega|\alpha|^{2} \Omega^{2} /(\Delta \theta)^{2}$ diverges for $\Delta \theta \downarrow 0$, because we need a not vanishing atom-field interaction in the limit. In the following we shall need the relation

$$
\left\langle j, m ; \varpi \mid \lambda_{+}\right\rangle=-\varpi \sqrt{\frac{2 j+1}{12}} \delta_{m, 1}
$$

The choice of a circular polarization is done in some experiments, because in this way, in the long run, only two states are involved in the dynamics (23. p. 206) and the usual theory has been developed for two-states systems [15, 16]; we shall see that also in our modified model the right circular polarization pushes the atom in the states $\left|F_{-}, F_{-}\right\rangle,\left|F_{+}, F_{+}\right\rangle$.

2.3.2. The equilibrium state. By a stochastic unitary transformation, we obtain from (14) and (20) an atomic density matrix

$$
\begin{aligned}
\hat{\rho}(t)=\exp \left\{\frac{\mathrm{i}}{2}\right. & \left.(\omega t+\sqrt{B} W(t))\left(P_{+}-P_{-}\right)\right\} \rho(t ; f) \\
& \times \exp \left\{-\frac{\mathrm{i}}{2}(\omega t+\sqrt{B} W(t))\left(P_{+}-P_{-}\right)\right\},
\end{aligned}
$$

which satisfies the "Ito's" master equation

$$
\mathrm{d} \hat{\rho}(t)=\mathcal{L}_{B}[\hat{\rho}(t)] \mathrm{d} t+\frac{\mathrm{i}}{2} \sqrt{B}\left[P_{+}-P_{-}, \hat{\rho}(t)\right] \mathrm{d} W(t)
$$

and whose mean value satisfies the ordinary master equation

$$
\rho(t)=\mathbb{E}[\hat{\rho}(t)], \quad \frac{\mathrm{d}}{\mathrm{d} t} \rho(t)=\mathcal{L}_{B}[\rho(t)] .
$$

The time independent Liouvillian entering equations (26) and (27) is given by

$\mathcal{L}_{B}[\rho]=\mathcal{L}_{0}[\rho]+\frac{B}{8}\left(\left[\left(P_{+}-P_{-}\right) \rho, P_{+}-P_{-}\right]+\left[P_{+}-P_{-}, \rho\left(P_{+}-P_{-}\right)\right]\right)$, 


$$
\begin{aligned}
& \mathcal{L}_{0}[\rho]=-\mathrm{i}[\widehat{H}, \rho]+\frac{1}{2} \sum_{k}\left(\left[D\left(h_{k}\right) \rho, D\left(h_{k}\right)^{\dagger}\right]+\left[D\left(h_{k}\right), \rho D\left(h_{k}\right)^{\dagger}\right]\right), \\
& \widehat{H}=\frac{1}{2}\left(\omega_{0}-\omega\right)\left(P_{+}-P_{-}\right)+\frac{\mathrm{i}}{2} \sum_{k}\left(\left\langle\left(S^{-}+\mathbb{1}\right) \lambda \mid h_{k}\right\rangle R_{k}-\left\langle h_{k} \mid\left(S^{-}+\mathbb{1}\right) \lambda\right\rangle R_{k}^{\dagger}\right) \\
&+\frac{\mathrm{i}}{2}\left(\left\langle\lambda \mid S^{+} \lambda\right\rangle-\left\langle S^{+} \lambda \mid \lambda\right\rangle\right) P_{+}+\frac{\mathrm{i}}{2}\left(\left\langle\lambda \mid S^{-} \lambda\right\rangle-\left\langle S^{-} \lambda \mid \lambda\right\rangle\right) P_{-},
\end{aligned}
$$

where $D(h)$ is a kind of effective dipole operator

$$
\begin{aligned}
D(h) & =\sum_{k}\left\langle h \mid h_{k}\right\rangle R_{k}+\left\langle h \mid\left(S^{+}-11\right) \lambda\right\rangle P_{+}+\left\langle h \mid\left(S^{-}-1\right) \lambda\right\rangle P_{-} \\
& =\alpha \sum_{m=-1}^{1}\langle h \mid 1, m ;+1\rangle A_{m}+\alpha \Omega \mathrm{e}^{\mathrm{i} \delta} \sum_{\epsilon= \pm}\left\langle h \mid\left(S^{\epsilon}-11\right) \lambda_{+}\right\rangle P_{\epsilon} .
\end{aligned}
$$

Now, let us set

$$
\begin{aligned}
& P_{\perp}=1-|1,1 ;+1\rangle\langle 1,1 ;+1|, \quad \varepsilon=\operatorname{Im}\left\langle S^{+} \lambda_{+} \mid P_{\perp} S^{-} \lambda_{+}\right\rangle, \\
& g_{ \pm}=P_{\perp}\left(S^{ \pm}-1\right) \lambda_{+}, \quad \Delta g=g_{+}-g_{-}, \\
& \delta_{ \pm}=\delta_{ \pm}(1 ;+1), \quad s=\delta_{+}-\delta_{-},
\end{aligned}
$$

We have to ask $|\varepsilon|<+\infty,\left\|g_{ \pm}\right\|<+\infty$, for $\Delta \theta \downarrow 0$; roughly speaking, $S_{ \pm}$must introduce small corrections even when the norm of $\lambda_{+}$diverges. Then, we have

$$
\begin{gathered}
P_{+} \mathcal{L}_{B}[\rho] P_{+}=-|\alpha|^{2} P_{+} \rho P_{+}-L^{-\dagger} P_{-} \rho P_{+}-P_{+} \rho P_{-} L^{-} \\
P_{-} \mathcal{L}_{B}[\rho] P_{-}=|\alpha|^{2} \sum_{m=-1}^{1} A_{m} P_{+} \rho P_{+} A_{m}^{\dagger}+L^{-} P_{+} \rho P_{-}+P_{-} \rho P_{+} L^{-\dagger} \\
P_{-} \mathcal{L}_{B}[\rho] P_{+}=\left(P_{+} \mathcal{L}_{B}\left[\rho^{\dagger}\right] P_{-}\right)^{\dagger}=L^{+} P_{+} \rho P_{+}-P_{-} \rho P_{-} L^{-} \\
+\left[\mathrm{i}\left(\omega_{0}-\omega+\operatorname{Im}\left\langle S^{+} \lambda \mid S^{-} \lambda\right\rangle\right)-\frac{1}{2}\left(|\alpha|^{2}+B+\left\|\left(S^{+}-S^{-}\right) \lambda\right\|^{2}\right)\right] P_{-} \rho P_{+} \\
L^{ \pm}=\sum_{k}\left\langle S^{ \pm} \lambda \mid h_{k}\right\rangle R_{k}=-\frac{1}{2}|\alpha|^{2} \Omega \mathrm{e}^{-\mathrm{i}\left(\delta+2 \delta_{ \pm}\right)} A_{1} \\
\left(S^{ \pm}-1\right) \lambda_{+}=-\mathrm{ie}^{\mathrm{i} \delta_{ \pm}} \sin \delta_{ \pm}|1,1 ;+1\rangle+g_{ \pm} \\
\operatorname{Im}\left\langle S^{+} \lambda \mid S^{-} \lambda\right\rangle=|\alpha|^{2} \Omega^{2}\left(\varepsilon-\frac{1}{4} \sin 2 s\right) \\
\left\|\left(S^{+}-S^{-}\right) \lambda\right\|^{2}=|\alpha|^{2} \Omega^{2}\left(\sin ^{2} s+\|\Delta g\|^{2}\right)
\end{gathered}
$$

Then, one can check that the equilibrium state $\rho_{\infty}$ for the master equation (27) is supported by the two extreme states

$$
|1\rangle=\left|F_{+}, F_{+}\right\rangle, \quad|2\rangle=\left|F_{-}, F_{-}\right\rangle .
$$

By using these two states the density matrix $\rho_{\infty}$ is given by

$$
\begin{aligned}
& \rho_{\infty}=\left(\begin{array}{cc}
\Omega^{2} d_{1} & \Omega \exp \left[\mathrm{i}\left(\delta+2 \delta_{-}\right)\right] d_{2} \\
\Omega \exp \left[-\mathrm{i}\left(\delta+2 \delta_{-}\right)\right] d_{3} & 1-\Omega^{2} d_{1}
\end{array}\right), \\
& \boldsymbol{d}=\left(\begin{array}{c}
d_{1} \\
d_{2} \\
d_{3}
\end{array}\right), \quad \boldsymbol{G d}=\boldsymbol{w}, \quad \boldsymbol{w}=\frac{1}{2}\left(\begin{array}{l}
0 \\
1 \\
1
\end{array}\right)
\end{aligned}
$$


Quantum stochastic models of two-level atoms

$$
\begin{aligned}
& \boldsymbol{G}=\left(\begin{array}{ccc}
1 & -1 / 2 & -1 / 2 \\
\Omega^{2} \mathrm{e}^{\mathrm{i} s} \cos s & b & 0 \\
\Omega^{2} \mathrm{e}^{-\mathrm{i} s} \cos s & 0 & \bar{b}
\end{array}\right), \\
& b=\frac{1}{2}\left[1+y+\Omega^{2}\left(\|\Delta g\|^{2}+\sin ^{2} s\right)\right]-\mathrm{i}\left(\widetilde{z}+\frac{1}{4} \Omega^{2} \sin 2 s\right), \\
& \widetilde{z}=z-\Omega^{2} \varepsilon, \quad z=\left(\omega-\omega_{0}\right) /|\alpha|^{2}, \quad y=B /|\alpha|^{2} .
\end{aligned}
$$

It turns out

$$
\begin{gathered}
d_{1}=\operatorname{Re} d, \quad d_{2}=d, \quad d_{3}=\bar{d}, \\
d=\frac{1+y+\Omega^{2}\left(\|\Delta g\|^{2}+\sin ^{2} s\right)+\mathrm{i}\left(2 \widetilde{z}-\Omega^{2} \sin s \cos s\right)}{4 \widetilde{z}^{2}+\Gamma^{2}}, \\
\Gamma^{2}=\left(1+y+\Omega^{2}\|\Delta g\|^{2}\right)^{2}+\Omega^{2}\left(2+2 y+2 \Omega^{2}\|\Delta g\|^{2}+\Omega^{2} \sin ^{2} s\right) .
\end{gathered}
$$

\section{The fluorescence spectrum}

\subsection{Heterodyne detection}

3.1.1. The output current. A way to obtain the spectrum of the stimulated atom is by means of balanced heterodyne detection; in such a scheme the light emitted by the atom is made to interfere with the light of a strong monochromatic laser (the local oscillator) and then detected by a couple of photocounters [28]. A mathematical description of such a detection scheme has been given inside the theory of continuous measurements [12, 29]; the output current of the detector is represented by the operator

$I(\nu, h ; t)=\int_{0}^{t} F(t-s) \mathrm{e}^{\mathrm{i} \nu s} \mathrm{~d} A(h ; s)+$ h.c.,$\quad \mathrm{d} A(h ; t)=\sum_{k}\left\langle h \mid h_{k}\right\rangle \mathrm{d} A_{k}(t)$,

where $F(t)$ is the detector response function, say

$$
F(t)=k_{1} \sqrt{\frac{\widetilde{\gamma}}{4 \pi}} \exp \left(-\frac{\widetilde{\gamma}}{2} t\right), \quad \widetilde{\gamma}>0,
$$

$k_{1} \neq 0$ has the dimensions of a current, $\nu$ is the frequency of the local oscillator and $h \in \mathcal{Z},\|h\|=1$. Any information on the localization and on the polarization of the detector is contained in $h$. We assume that the detector spans a small solid angle, so that $h$ is given by

$$
h\left(\theta^{\prime}, \phi^{\prime}\right)=\frac{1_{\Delta \Upsilon}\left(\theta^{\prime}, \phi^{\prime}\right)}{\sqrt{|\Delta \Upsilon|}} \vec{e}\left(\theta^{\prime}, \phi^{\prime}\right)
$$

where $\Delta \Upsilon$ is a small solid angle around the direction $(\theta, \phi), \Delta \Upsilon \downarrow\{(\theta, \phi)\},|\Delta \Upsilon| \simeq$ $\sin \theta \mathrm{d} \theta \mathrm{d} \phi$ and $\vec{e}$ is a complex polarization vector, $\left|\vec{e}\left(\theta^{\prime}, \phi^{\prime}\right)\right|=1$. Moreover, we assume that the transmitted wave does not reach the detector, i.e. $\theta>0$ and so

$$
\langle h \mid \lambda\rangle=0 .
$$

From the canonical commutation relations for the fields one has that $I\left(\nu, h ; t_{1}\right)$ and $I\left(\nu, h ; t_{2}\right)$ are compatible observables for any choice of the times; the same holds in the Heisenberg picture. Then, a theory of continuous observation of $I(\nu, h ; t), t \geq 0$, can be developed [12, 14] and a probability law for the stochastic process "output 
current" can be constructed; however, to obtain the spectrum we do not need the full theory, but only the second moment of $I$. Here and in the following for the expectation of any operator $M$ we shall use the notation

$$
\langle\langle M\rangle\rangle=\mathbb{E}\left[\operatorname{Tr}_{\mathcal{H} \otimes \mathcal{F}}\left\{M\left(\rho_{0} \otimes|e(f)\rangle\langle e(f)|\right)\right\}\right] .
$$

3.1.2. The power spectrum. In the long run the mean of the output power is given by

$$
P(\nu, h)=\lim _{T \rightarrow+\infty} \frac{k_{2}}{T} \int_{0}^{T}\left\langle\left\langle U(T)^{\dagger}(I(\nu, h ; t))^{2} U(T)\right\rangle\right\rangle \mathrm{d} t ;
$$

$k_{2}>0$ has the dimensions of a resistance, it is independent of $\nu$, but it can depend on the other features of the detection apparatus. As a function of $\nu, P(\nu, h)$ gives the power spectrum observed in the "channel $h$ "; in the case of the choice (44) it is the spectrum observed around the direction $(\theta, \phi)$ and with polarization $\vec{e}$.

The mean power $P(\nu, h)$, under condition (45), can be expressed as

$$
\begin{gathered}
P(\nu, h)=\frac{k_{1}^{2} k_{2}}{4 \pi}+k_{1}^{2} k_{2} \Sigma(x ; h), \\
\Sigma(x ; h)=\frac{\Omega^{2}}{2 \pi} \int_{0}^{+\infty} \mathrm{d} t \mathrm{e}^{-q t} \operatorname{Tr}_{\mathcal{H}}\left\{\widetilde{D}(h)^{\dagger} \mathrm{e}^{\mathcal{K} t}\left[\widetilde{D}(h) \rho_{\infty}\right]\right\}+\text { c.c. }, \\
x=\frac{\nu-\omega_{0}}{|\alpha|^{2}}, \quad \gamma=\frac{\widetilde{\gamma}}{|\alpha|^{2}}, \quad q=\frac{\gamma+y}{2}+\mathrm{i}(x-z) . \\
\widetilde{D}(h)=\mathrm{e}^{-\mathrm{i} \delta} D(h) /(\alpha \Omega), \\
|\alpha|^{2} \mathcal{K}[\rho]=\mathcal{L}_{B}[\rho]-B P_{+} \rho P_{-}+B P_{-} \rho P_{+} ;
\end{gathered}
$$

$D(h)$ is given by equation (29),$\rho_{\infty}$ by (34), $\mathcal{L}_{B}$ by (31a) $-(31 d), y$ and $z$ by (38).

In the expression (48) of the power the term $k_{1}^{2} k_{2} /(4 \pi)$ appears, independent of $\nu$; it is a white noise contribution due to the detection scheme, coming out mathematically from the canonical commutation relations for the fields and known as "shot noise". The semigroup $\exp [\mathcal{K} t]$ is trace preserving, but not positivity preserving, while $\rho_{\infty}$ is the equilibrium state of $\mathcal{L}_{B}$, which is a bona-fide Liouvillian, because it can be written in the Lindblad form (28d), (28b).

3.1.3. Sketch of the proof of equations (48) and (49). The computations are similar to those of reference [19], but now not only QSC but also classical stochastic calculus plays a role. Here we give only the main intermediate steps; a more detailed account can be found in [20], where however the degeneracy of the levels is not considered.

The first step is to rearrange in normal order the field operators involved in (47); in this way the constant shot noise appears and the spectrum reduces to

$$
\begin{aligned}
\Sigma(x ; h) & =\lim _{T \rightarrow+\infty} \frac{1}{2 \pi T}\left\langle\left\langle\int_{0}^{T} \mathrm{~d} A_{\text {out }}^{\dagger}(h ; t) \int_{0}^{t} \mathrm{~d} A_{\text {out }}(h ; s) \mathrm{e}^{-\left(\frac{\tilde{\gamma}}{2}+\mathrm{i} \nu\right)(t-s)}\right\rangle\right\rangle+\text { c.c. } \\
& =\lim _{T \rightarrow+\infty} \frac{1}{2 \pi T}\left\langle\left\langle\int_{0}^{T} \mathrm{~d} A_{\text {out }}^{\dagger}(h ; t) \mathrm{e}^{-\mathrm{i} \nu t} \int_{0}^{T} \mathrm{~d} A_{\text {out }}(h ; s) \mathrm{e}^{\mathrm{i} \nu s} \mathrm{e}^{-\frac{\tilde{\gamma}}{2}|t-s|}\right\rangle\right\rangle \geq 0 ;(533
\end{aligned}
$$


the second expression shows the positivity of $\Sigma(x ; h)$, while the first one is useful for the successive developments. The output fields [12] are given by $A_{\text {out }}(h ; t)=$ $U(t)^{\dagger} A(h ; t) U(t)$ and we have

$$
\mathrm{d} A_{\text {out }}(h ; t)=\sum_{k}\left\langle h \mid h_{k}\right\rangle U(t)^{\dagger}\left(\sum_{l} S_{k l} \mathrm{~d} A_{l}(t)+R_{k} \mathrm{~d} t\right) U(t) .
$$

The second step is to show, by using (54), (25), (29), (45) and the factorization properties of Fock space, that

$$
\begin{aligned}
& \Sigma(x ; h)=\lim _{T \rightarrow+\infty} \frac{1}{2 \pi T} \int_{0}^{T} \mathrm{~d} t \int_{0}^{t} \mathrm{~d} s \exp \left\{-\left[\frac{\widetilde{\gamma}}{2}+\mathrm{i}(\nu-\omega)\right](t-s)\right\} \\
& \times \mathbb{E}\left[\operatorname { e x p } \{ \mathrm { i } \sqrt { B } [ W ( t ) - W ( s ) ] \} \operatorname { T r } _ { \mathcal { H } \otimes \mathcal { F } } \left\{D(h)^{\dagger} C(t, s) D(h)\right.\right. \\
& \left.\left.\times(\hat{\rho}(s) \otimes|e(f)\rangle\langle e(f)|) C(t, s)^{\dagger}\right\}\right]+ \text { c.c. }, \\
& C(t, s)=\exp \left\{\frac{\mathrm{i}}{2}[\omega t+\sqrt{B} W(t)]\left(P_{+}-P_{-}\right)\right\} U(t) U(s)^{\dagger} \\
& \times \exp \left\{-\frac{\mathrm{i}}{2}[\omega s+\sqrt{B} W(s)]\left(P_{+}-P_{-}\right)\right\} .
\end{aligned}
$$

Finally an interplay between classical stochastic calculus and the quantum regression theorem, which holds for a dynamic like $C(t, s)$, gives the expression (49).

3.1.4. The angular distribution of the spectrum. Let us stress that $\rho_{\infty}$ is supported by the two states $|1\rangle=\left|F_{+}, F_{+}\right\rangle,|2\rangle=\left|F_{-}, F_{-}\right\rangle$and that all the operations involved in (49) leave the span of $|1\rangle,|2\rangle$ invariant. So we are left with computations involving $2 \times 2$ matrices; from now on we suppress the index $\mathcal{H}$ in the trace and we use the Pauli matrices $\sigma_{ \pm}, \sigma_{z}, \ldots$ In particular we have $P_{ \pm}=\frac{1}{2}\left(1 \pm \sigma_{z}\right)$ and

$$
\begin{aligned}
& \widetilde{D}(h)=\langle h \mid 1,1 ;+1\rangle D_{1}+\left\langle h \mid g_{+}\right\rangle P_{+}+\left\langle h \mid g_{-}\right\rangle P_{-}, \\
& D_{1}=\frac{1}{\Omega} \mathrm{e}^{-\mathrm{i} \delta} \sigma_{-}-\mathrm{ie}^{\mathrm{i} \delta_{+}} \sin \delta_{+} P_{+}-\mathrm{ie} \mathrm{i}^{\mathrm{i} \delta_{-}} \sin \delta_{-} P_{-} .
\end{aligned}
$$

To obtain the angular dependence of the spectrum, let us introduce the states $h_{ \pm}$concentrated around $(\theta, \phi)$ and with right/left circular polarization, given by equation (44) with $\vec{e}=\vec{e}_{ \pm}$, where $\vec{e}_{ \pm}(\theta, \phi)^{\dagger}=\frac{\exp (-\mathrm{i} \phi)}{\sqrt{2}}(-\mathrm{i} \sin \phi \mp \cos \theta \cos \phi$, i $\cos \phi \mp$ $\cos \theta \sin \phi, \pm \sin \theta)$. Then, the two angular spectra $\Sigma_{ \pm}(x ; \theta)$, which, by the cylindrical symmetry of the problem, do not depend on $\phi$, are given by

$$
\begin{gathered}
\Sigma_{ \pm}(x ; \theta)=\frac{1}{|\Delta \Upsilon|} \Sigma\left(x ; h_{ \pm}\right)=\frac{\Omega^{2}}{2 \pi} \int_{0}^{+\infty} \mathrm{d} t \mathrm{e}^{-q t} \operatorname{Tr}\left\{D_{ \pm}(\theta)^{\dagger} \mathrm{e}^{\mathcal{K} t}\left[D_{ \pm}(\theta) \rho_{\infty}\right]\right\}+\text { c.c. } \\
D_{ \pm}(\theta)= \pm \frac{1}{4} \sqrt{\frac{3}{2 \pi}}(1 \pm \cos \theta) D_{1}+\sum_{\epsilon= \pm} g_{\epsilon}(\theta ; \pm) P_{\epsilon}
\end{gathered}
$$

The functions $g_{\epsilon}(\theta ; \pm)=\left\langle h_{ \pm} \mid g_{\epsilon}\right\rangle / \sqrt{|\Delta \Upsilon|}$ depend on $S^{\epsilon}$, but after all they are free parameters of the theory: they are square integrable $\theta$-functions, satisfying the constraint $\int_{0}^{\pi} \sin \theta\left[(1+\cos \theta) g_{\epsilon}(\theta ;+)-(1-\cos \theta) g_{\epsilon}(\theta ;-)\right] \mathrm{d} \theta=0$, coming from the orthogonality of $g_{\epsilon}$ to $|1,1 ;+1\rangle$, see $(30 b)$. 
Then, by integrating over the whole solid angle, one gets the total spectrum

$\Sigma(x)=\int_{0}^{\pi} \mathrm{d} \theta \sin \theta \int_{0}^{2 \pi} \mathrm{d} \phi\left(\Sigma_{+}(x ; \theta)+\Sigma_{-}(x ; \theta)\right)=\sum_{k} \Sigma\left(\nu ; h_{k}\right)$,

where $\left\{h_{k}\right\}$ is any c.o.n.s. in $\mathcal{Z}$. When one has $g_{\epsilon}=0$, as in the usual case, one gets

$$
\Sigma_{ \pm}(x ; \theta)=\frac{3}{8 \pi}\left(\frac{1 \pm \cos \theta}{2}\right)^{2} \Sigma(x) .
$$

For $g_{\epsilon} \neq 0$ the $x$ and $\theta$ dependencies do not factorize. In the experiments one measures something proportional to $\Sigma_{+}(x ; \theta)+\Sigma_{-}(x ; \theta)$ for $\theta$ around $\pi / 2$; this quantity fails to be proportional to $\Sigma(x)$ only by the presence of some terms which we expect to be small and which are not qualitatively different from the other terms in $\Sigma(x)$. So, for simplicity, we shall study only the total spectrum (61).

\subsection{The total spectrum}

By choosing in (61) a basis with $h_{1}=|1,1 ;+1\rangle, \quad h_{2}=\Delta g /\|\Delta g\|, \quad h_{3}=$ $\|\Delta g\|^{-1}\left[\left\|g_{-}\right\|^{2}\|\Delta g\|^{2}-\left|\left\langle\Delta g \mid g_{-}\right\rangle\right|^{2}\right]^{-1 / 2}\left[\|\Delta g\|^{2} g_{-}-\left\langle\Delta g \mid g_{-}\right\rangle \Delta g\right]$, we get

$$
\begin{gathered}
\sum_{k} \operatorname{Tr}\left\{\widetilde{D}\left(h_{k}\right)^{\dagger} \mathrm{e}^{\mathcal{K} t}\left[\widetilde{D}\left(h_{k}\right) \rho_{\infty}\right]\right\}=\operatorname{Tr}\left\{D_{1}^{\dagger} \mathrm{e}^{\mathcal{K} t}\left[D_{1} \rho_{\infty}\right]\right\}+\left\langle g_{-} \mid \Delta g\right\rangle \operatorname{Tr}\left\{P_{+} \rho_{\infty}\right\} \\
+\left\langle\Delta g \mid g_{-}\right\rangle \operatorname{Tr}\left\{P_{+} \mathrm{e}^{\mathcal{K} t}\left[\rho_{\infty}\right]\right\}+\left\|g_{-}\right\|^{2}+\|\Delta g\|^{2} \operatorname{Tr}\left\{P_{+} \mathrm{e}^{\mathcal{K} t}\left[P_{+} \rho_{\infty}\right]\right\} .
\end{gathered}
$$

Then, from (49), (61), (63), we get the final expression of the total spectrum

$$
\begin{aligned}
& \Sigma(x)=\frac{\Omega^{2}}{2 \pi}\left[\frac { 1 } { q } ( v _ { 3 } + \mathrm { ie } ^ { \mathrm { i } \delta _ { - } } \operatorname { s i n } \delta _ { - } + \mathrm { i } \Omega ^ { 2 } v _ { 1 } \mathrm { e } ^ { - \mathrm { i } s } \operatorname { s i n } s ) \left(d_{2}-\mathrm{ie}^{-\mathrm{i} \delta_{-}} \sin \delta_{-}\right.\right. \\
& \left.-\mathrm{i} \Omega^{2} d_{1} \mathrm{e}^{\mathrm{i} s} \sin s\right)+\mathrm{e}^{-\mathrm{i} \delta_{-}} \sin \delta_{+}\left(\Omega^{2} c_{1} \sin s-\mathrm{ie}^{\mathrm{i} s} c_{3}\right) \\
& +\left(d_{1}+\mathrm{i} d_{3} \mathrm{e}^{\mathrm{i} s} \sin s\right)\left(u_{3}+\mathrm{i} \Omega^{2} u_{1} \mathrm{e}^{-\mathrm{i} s} \sin s\right)+\frac{1}{q}\left\langle g_{-} \mid g_{-}+\Omega^{2} d_{1} \Delta g\right\rangle \\
& \left.+\Omega^{2}\left\langle\Delta g \mid g_{+}\right\rangle c_{1}-\Omega^{2} d_{3}\|\Delta g\|^{2} u_{1}+\frac{\Omega^{2}}{q}\left\langle\Delta g \mid g_{-}+\Omega^{2} d_{1} \Delta g\right\rangle v_{1}+\text { c.c. }\right], \\
& \boldsymbol{v}=\frac{1}{\boldsymbol{K}+q} \boldsymbol{w}, \quad \boldsymbol{c}=\frac{1}{\boldsymbol{K}+q} \boldsymbol{d}, \quad \boldsymbol{u}=\frac{1}{\boldsymbol{K}+q}\left(\begin{array}{c}
0 \\
0 \\
1
\end{array}\right), \\
& \boldsymbol{K}=\boldsymbol{G}+y\left(\begin{array}{ccc}
0 & 0 & 0 \\
0 & 1 & 0 \\
0 & 0 & -1
\end{array}\right)
\end{aligned}
$$

By integrating over the reduced frequency $x$, we get the strength of the spectrum

$$
\begin{aligned}
\int_{-\infty}^{+\infty} \Sigma(x) \mathrm{d} x= & \Omega^{2}\left\{d_{1}\left(1+\Omega^{2} \sin ^{2} \delta_{+}\right)+\left(1-\Omega^{2} d_{1}\right)\left(\sin ^{2} \delta_{-}+\left\|g_{-}\right\|^{2}\right)\right. \\
& \left.+\operatorname{Re}\left[d_{2}\left(\mathrm{e}^{2 \mathrm{i} \delta_{-}}-1\right)\right]+\Omega^{2} d_{1}\left\|g_{+}\right\|^{2}\right\} .
\end{aligned}
$$

Let us recall that $|\alpha|^{2}$ is the natural line width, $\Omega^{2}$ is proportional to the laser intensity, $z=\left(\omega-\omega_{0}\right) /|\alpha|^{2}$ is the reduced detuning, to which also the parameter $\widetilde{z}=z-\Omega^{2} \varepsilon$ is linked, $y=B /|\alpha|^{2}$ is the reduced laser bandwidth (38), $\Omega^{2}|\alpha|^{2} \varepsilon$ is an intensity dependent shift, $x=\left(\nu-\omega_{0}\right) /|\alpha|^{2}$ and $\gamma=\widetilde{\gamma} /|\alpha|^{2}$ are the reduced frequency 
and the reduced instrumental width, respectively, and $q=\mathrm{i}(x-z)+(\gamma+y) / 2(50)$. The other quantities entering all these equations are given by the equations (30b), (30), (35)-(41); in particular, $s=\delta_{+}-\delta_{-}$and $\Delta g=g_{+}-g_{-}$. Let us note that $\varepsilon, \delta_{ \pm},\left\|g_{ \pm}\right\|^{2},\left\langle g_{+} \mid g_{-}\right\rangle$are parameters linked to the $S^{ \pm}$scattering matrices, satisfying the constraints " $\left|\left\langle g_{+} \mid g_{-}\right\rangle\right| \leq\left\|g_{+}\right\|\left\|g_{-}\right\| "$ and " $\|\Delta g\|=0 \Rightarrow \varepsilon=0 "$ (apart from this relation $\varepsilon$ is an independent parameter of the model). One can check that the spectrum $\Sigma(x)$ is invariant under the transformation: $x \rightarrow-x, z \rightarrow-z, \varepsilon \rightarrow-\varepsilon$, $\delta_{ \pm} \rightarrow-\delta_{ \pm},\left\langle g_{-} \mid g_{+}\right\rangle \rightarrow\left\langle g_{+} \mid g_{-}\right\rangle$.

3.2.1. The case of a monochromatic laser, $y=0$. When the laser bandwidth vanishes the spectrum (64) reduces to the one obtained in [19], where the polarization of light and the level degeneracy were not considered; if also $S^{ \pm}=11$ is taken, it reduces to the Mollow spectrum [15. A particularity of the $y=0$ case is that the spectrum can be decomposed into an elastic and an inelastic part, which in turn are proportional to the elastic and inelastic electromagnetic cross sections, as explained in [19]; for $y>0$ such a decomposition has no meaning. Figures 2 and 3 of [19] compare the spectra (for $\gamma=0.6$ and for various detunings and laser intensities) in the Mollow case and in the modified case with $\delta_{+}=-0.03, \delta_{-}=0.13,\left\|g_{ \pm}\right\|^{2}=0.005,\left\langle g_{+} \mid g_{-}\right\rangle=-0.005$, $\varepsilon=-0.001$; the two figures have $x-z$ in the horizontal axis and $\Sigma / \Omega^{2}$ in the vertical one. The parameters have been chosen in such a way that the modified spectrum would not too different from the Mollow one in the resonant case. In plotting the spectrum a factor $1 / 2$ has been inserted by error in the inelastic part, so the two figures have only a qualitative meaning; in any case, one sees the symmetry of the Mollow spectrum which is lost in the modified case.

3.2.2. The case $S^{ \pm}=1$. Let us recall that the usual model, with only the absorption/emission process, corresponds to $\delta_{ \pm}=0, g_{ \pm}=0, \varepsilon=0, z=\widetilde{z}, s=0$. In this case we obtain

$$
\begin{aligned}
& \Sigma(x)=\frac{\Omega^{2}}{2 \pi}\left[\frac{1}{q} v_{3} d+\left(2 v_{3}+\frac{4 \Omega^{2}}{N}\right) \operatorname{Re} d\right]+\text { c.c. }, \\
& d=\frac{1+y+2 \mathrm{i} z}{4 z^{2}+\Gamma^{2}}, \quad \Gamma^{2}=(1+y)\left(1+y+2 \Omega^{2}\right), \\
& v_{3}=[2+\gamma+y+2 \mathrm{i}(x-z)][1+\gamma+4 y+2 \mathrm{i}(x-2 z)] / N, \\
& N=4 \Omega^{2}[1+\gamma+2 y+2 \mathrm{i}(x-z)]+[2+\gamma+y+2 \mathrm{i}(x-z)] \\
& \quad \times[1+\gamma+4 y+2 \mathrm{i}(x-2 z)](1+\gamma+2 \mathrm{i} x) .
\end{aligned}
$$

Now, the spectrum $\Sigma(x)$ is invariant under the transformation: $x \rightarrow-x, z \rightarrow-z$.

If we put also $\gamma=0$, which means that the instrumental width is negligible, then one can check that the fluorescence spectrum $\Sigma(x)$ coincides exactly (apart from the different normalization) with the spectrum computed by Kimble and Mandel [16, 17]; when also the laser bandwidth $y$ vanishes, the equations above reduce once again to the Mollow spectrum [15]. One of the more interesting results of Kimble and Mandel is that a not vanishing laser bandwidth renders asymmetric the Mollow spectrum and enhances the side peak near $\omega_{0}$, as shown in the figures of $|16|$. In our notations, the figures 2, 3, 4 of 16 give, for $\gamma=0, \pi \Sigma$ on the z-axis versus $2(x-z)$ on the x-axis and $y$ on the y-axis; figure 2 is for $\Omega^{2}=0.0025$ and $z=-2.5$, figure 3 for $\Omega^{2}=25$ 

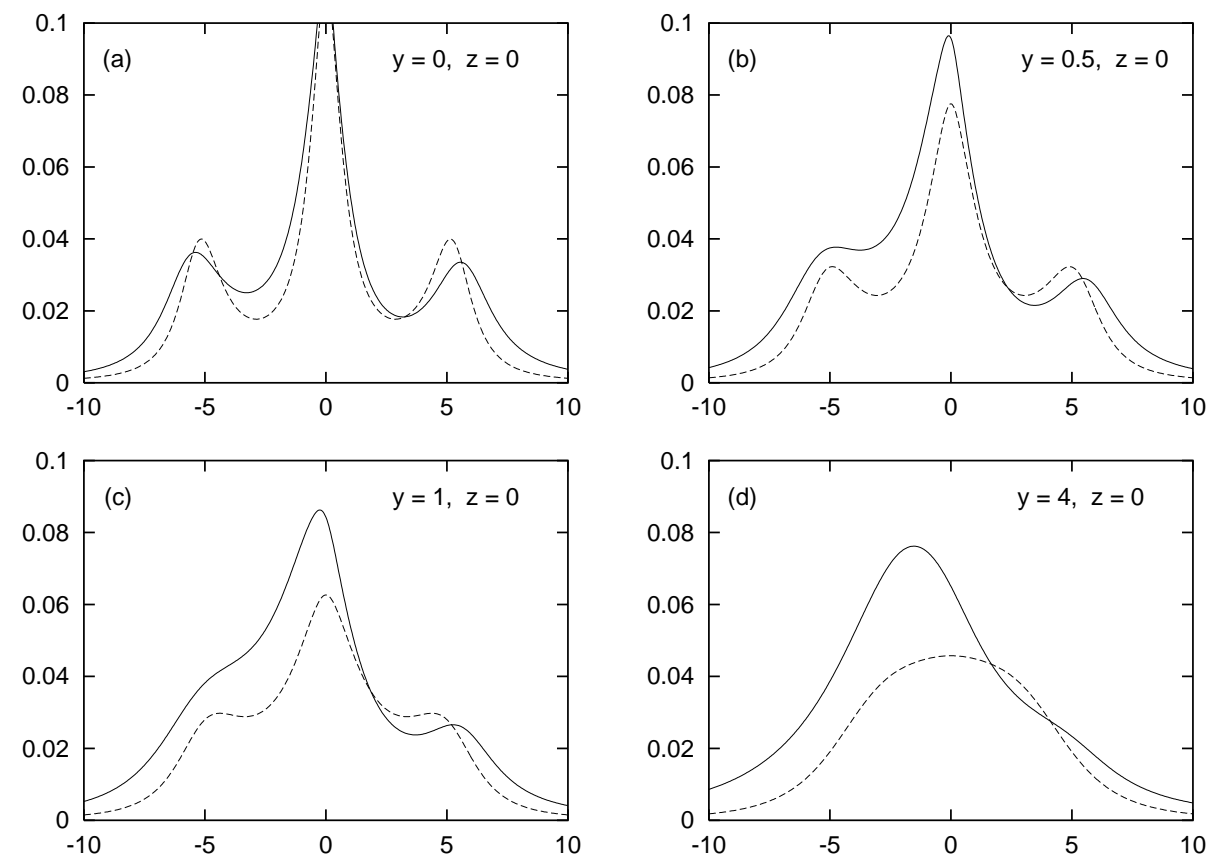

Figure 1. The spectrum $\Sigma(x)$ for $\gamma=0.6, \Omega^{2}=28, z=0$ and laser bandwidths $y=0,0.5,1,4$. Solid lines: modified model with the parameters given in section 3.2.5; dashed lines: usual model.

and $z=0$, figure 4 for $\Omega^{2}=25$ and $z=-1.5$. The figures 5 and 6 of 16 give, for $\gamma=0, \pi \Sigma$ on the $\mathrm{z}$-axis versus $2(x-z)$ on the $\mathrm{x}$-axis and $2 z$ on the $\mathrm{y}$-axis; figure 5 is for $\Omega^{2}=25, y=0.5$ and figure 6 is for $\Omega^{2}=2500, y=10$.

3.2.3. Low intensity laser. For a laser of low intensity the spectrum reduces to

$$
\begin{aligned}
&\left.\frac{\pi \Sigma(x)}{2 \Omega^{2}}\right|_{\Omega^{2}=0}=(\gamma+y)|c(x-z, \gamma+y)|^{2}\left(\left\|g_{-}\right\|^{2}+\frac{\gamma}{\gamma+y}|a(y, z)|^{2}\right) \\
&+y|c(x-z, \gamma+y) a(y, z)+c(-x, 1+\gamma) c(-z, 1+y)|^{2} \\
&+\gamma y|c(-x, 1+\gamma)|^{2}|c(-z, 1+y)|^{2}, \\
& a(y, z)=c(-z, 1+y)-\mathrm{ie}^{-\mathrm{i} \delta_{-}} \sin \delta_{-}, \quad c(x, \Delta)=\frac{1}{\Delta+2 \mathrm{i} x} .
\end{aligned}
$$

In this case one sees well why a decomposition in elastic and inelastic parts is not possible for $y>0$. Indeed, one has two peaks, one centered at $\nu=\omega$ (laser frequency) and one centered at $\nu=\omega_{0}$ (atomic frequency); the first peak can be interpreted as coming from an elastic channel and the second one as coming from an inelastic channel, but the second term in the r.h.s. of $(72)$ gives rise to an interference term between the two channels. 

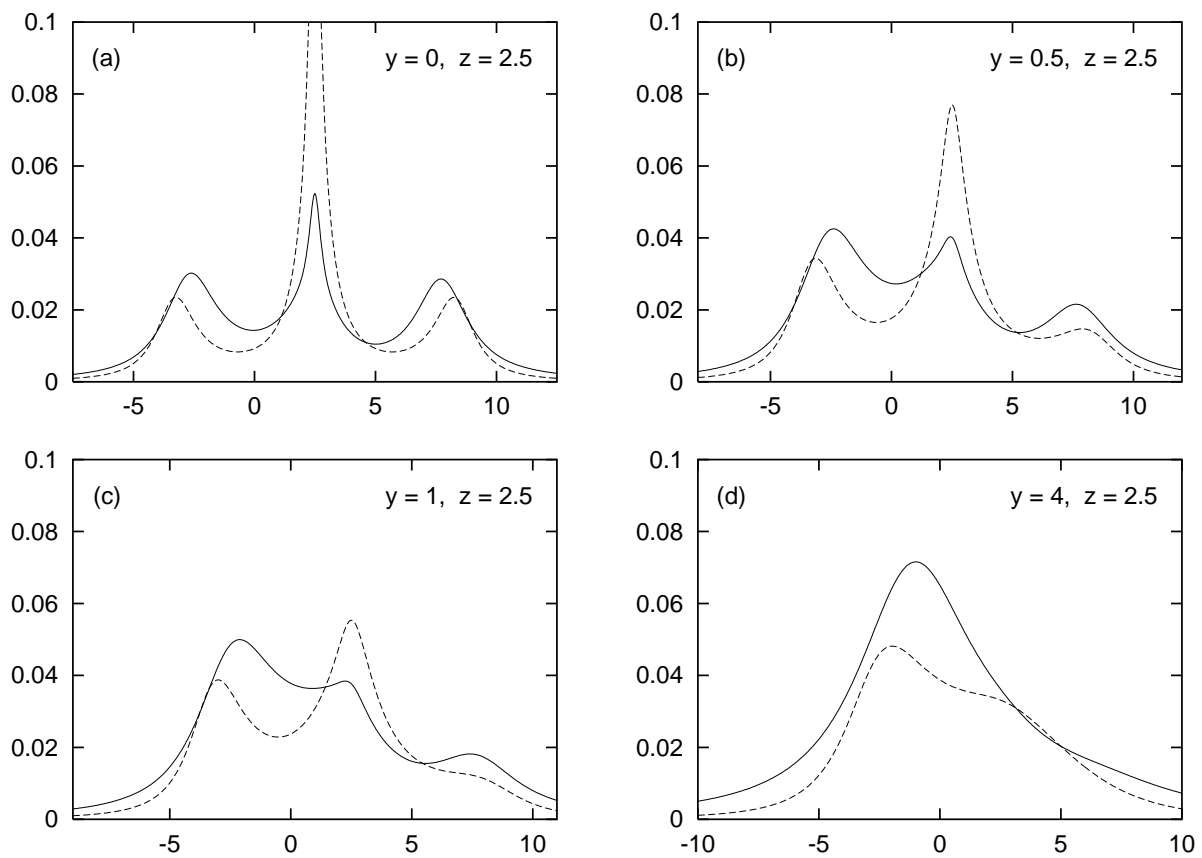

Figure 2. The spectrum $\Sigma(x)$ for $\gamma=0.6, \Omega^{2}=28, z=2.5$ and laser bandwidths $y=0,0.5,1,4$. Solid lines: modified model with the parameters given in section 3.2.5; dashed lines: usual model.

3.2.4. The broadband case. For a very large bandwidth, $y \gg x, z, \Omega^{2}$, the spectrum becomes independent from the detuning and reduces to

$\lim _{y \rightarrow \infty} \frac{\pi y}{2 \Omega^{2}} \Sigma(x)=\left|c(x-\eta, 1+\kappa)+\mathrm{i} \mathrm{e}^{\mathrm{i} \delta_{-}} \sin \delta_{-}\right|^{2}+\kappa|c(x-\eta, 1+\kappa)|^{2}+\left\|g_{-}\right\|^{2}$,

where $c(x, \Delta)$ is given in $(73)$ and

$$
\eta=\Omega^{2}\left(\varepsilon-\frac{1}{4} \sin 2 s\right), \quad \kappa=\gamma+\Omega^{2}\left(\sin ^{2} s+\|\Delta g\|^{2}\right) .
$$

Note the $\Omega$-dependent position of the peak, which can be interpreted as a light shift. In the usual case $\Sigma(x)$ becomes purely Lorentzian, centered in zero and with $\Omega$ independent width:

$$
\lim _{y \rightarrow \infty} \frac{\pi y}{2 \Omega^{2}} \Sigma(x)=\frac{1+\gamma}{(1+\gamma)^{2}+4 x^{2}} .
$$

3.2.5. Plots. In the general case the total spectrum is given by equations (64)-(66) In figures 1, 2 and 3 we compare the spectrum predicted by the usual model (dashed lines), in which only the absorption/emission channel is present, with the spectrum predicted by our modified model (solid lines), in which both the absorption/emission channel and the direct scattering channel are present. In both models we take $\gamma=0.6$, $\Omega^{2}=28$; the usual model is characterized by $\delta_{ \pm}=0,\left\|g_{ \pm}\right\|^{2}=0, \varepsilon=0$, while as an example of modified model we choose $\delta_{+}=-0.03, \delta_{-}=0.13,\left\|g_{+}\right\|^{2}=0.0045$, $\left\|g_{-}\right\|^{2}=0.0055,\left\langle g_{+} \mid g_{-}\right\rangle=-0.004+\mathrm{i} \times 0.002, \varepsilon=-0.001$. We consider three laser 

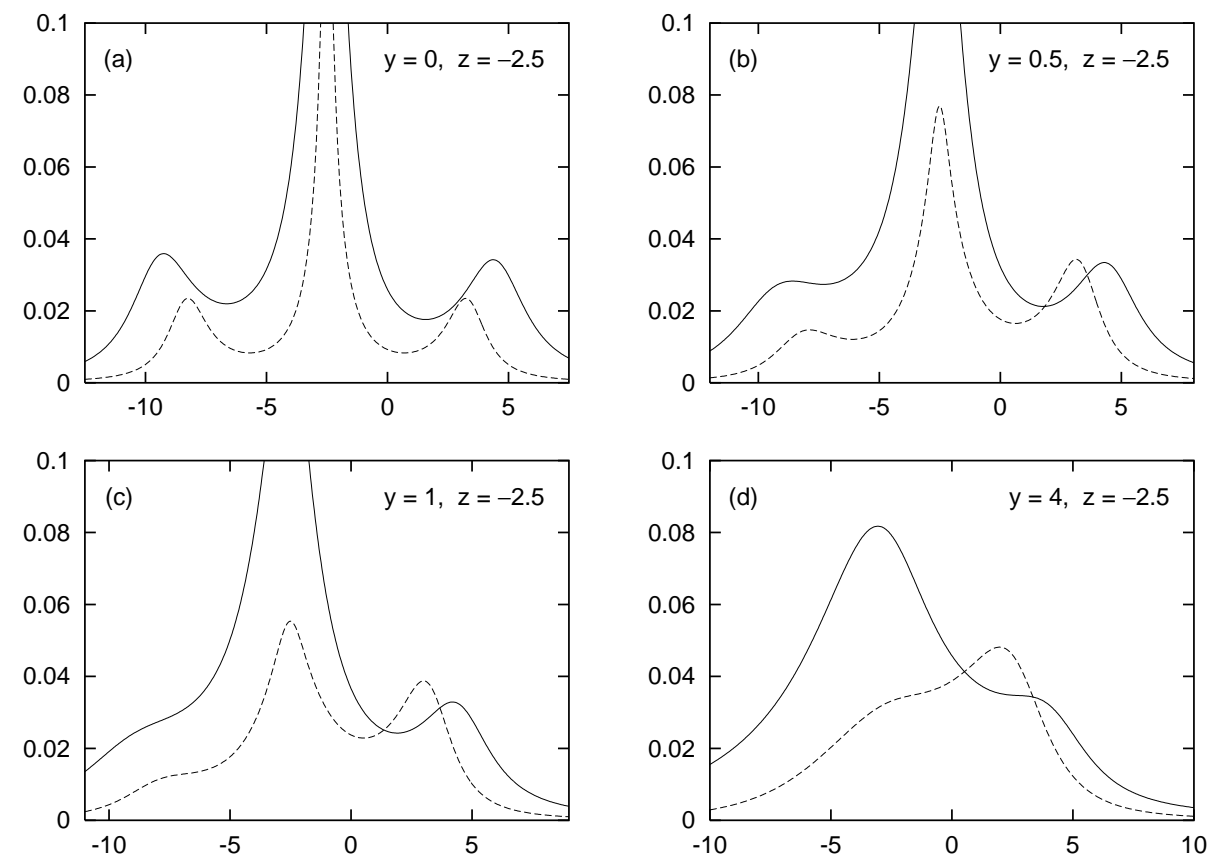

Figure 3. The spectrum $\Sigma(x)$ for $\gamma=0.6, \Omega^{2}=28, z=-2.5$ and laser bandwidths $y=0.0 .5,1,4$. Solid lines: modified model with the parameters given in section 3.2.5; dashed lines: usual model.

detunings $z=0,2.5,-2.5$ and four laser bandwidths $y=0,0.5,1,4$. Our choice of the parameters for the modified model is such that for a monochromatic laser in resonance (figure 1(a)) the modified spectrum is not quantitatively too different from the usual one, but its asymmetry is clear. The differences between the two cases are enhanced by the presence of both the detuning and the bandwidth.

Finally in figure 4 we consider a large bandwidth; however, $y$ is not much larger than $\Omega^{2}$, so that we are not exactly in the case of section 3.2.4. Anyway, only a peak survives and the spectrum is nearly independent from the detuning. In the usual case we see a nearly Lorentzian shape, centered at $x=0\left(\nu\right.$ near the atomic frequency $\left.\omega_{0}\right)$ and with a very weak residual $\Omega$-dependence. In the modified case the spectrum is strongly asymmetric and the position of the peak and the width are $\Omega$-dependent.

\section{References}

[1] Hudson R L and Parthasarathy K R 1984 Commun. Math. Phys. 93 301-23

[2] Gardiner C W and Collet M J 1985 Phys. Rev. A 31 3761-74

[3] Gardiner C W 1991 Quantum Noise (Berlin: Springer)

[4] Parthasarathy K R 1992 An Introduction to Quantum Stochastic Calculus (Basel: Birkhäuser)

[5] Gardiner C W 1986 Phys. Rev. Lett. 56 1917-20

[6] Barchielli A 1987 J. Phys. A: Math. Gen. 20 6341-55

[7] Kennedy T and Walls D F 1988 Phys. Rev. A 37 152-7

[8] Alsing P, Milburn G J and Walls D F 1988 Phys. Rev. A 37 2970-8

[9] Lane A S, Reid M D and Walls D F 1988 Phys. Rev. A 38 788-99

[10] Marte M A, Ritsch H and Walls D F 1988 Phys. Rev. A 38 3577-88 
(a) $\Omega^{2}=20$, usual model

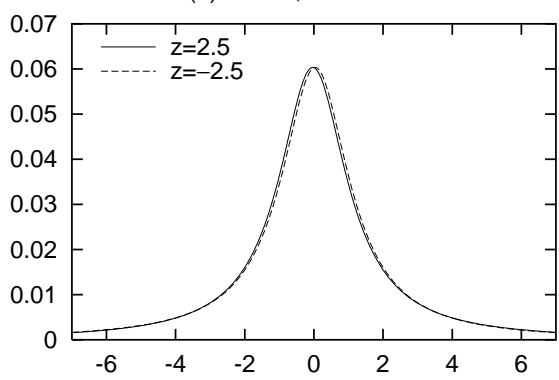

(c) $\Omega^{2}=40$, usual model

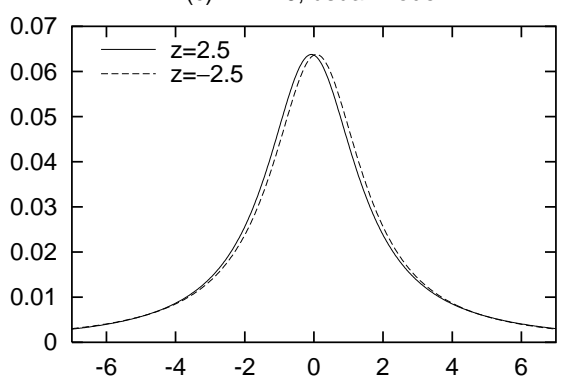

(b) $\Omega^{2}=20$, modified model

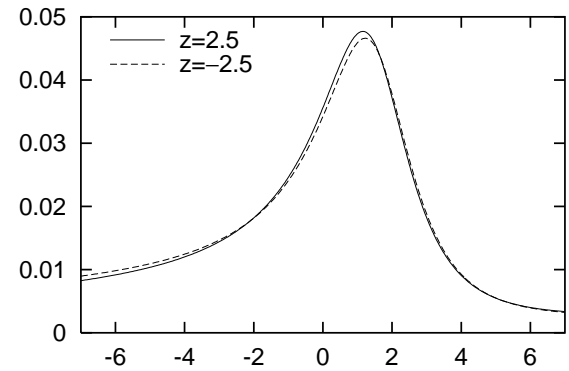

(d) $\Omega^{2}=40$, modified model

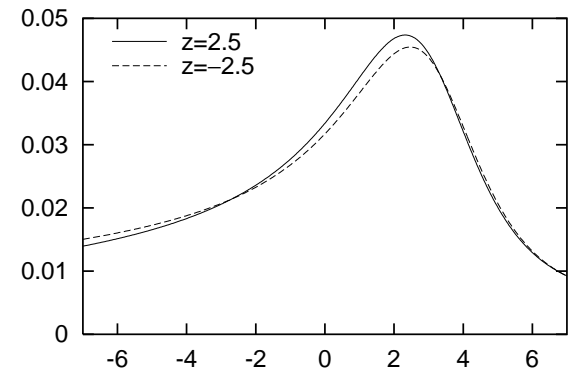

Figure 4. The spectrum $\Sigma(x)$ for laser bandwidth $y=50$ and $\gamma=0.6$, $\Omega^{2}=20,40, z=2.5,-2.5$. The parameters of the modified model are given in section 3.2.5.

[11] Collet M J and Walls D F 1988 Phys. Rev. Lett. 61 2442-4

[12] Barchielli A 1990 Quantum Opt. 2 423-41

[13] Wiseman H M and Milburn G J 1994 Phys. Rev. A 49 4110-25

[14] Barchielli A and Paganoni A M 1996 Quantum Semiclass. Opt. 8 133-56

[15] Mollow B R 1969 Phys. Rev. 188 1969-75

[16] Kimble H J and Mandel L 1977 Phys. Rev. A 15 689-99

[17] Kimble H J and Mandel L 1978 Multiphoton Processes edited by Eberly J H and Lambropoulos P (New York: Wiley) p 119-28

[18] Barchielli A and Lupieri G 1998 Quantum Probability, Banach Center Publications, Vol. 43 edited by Alicki R, Bozejko M and Majewski W A (Warsawa: Polish Academy of Sciences, Institute of Mathematics) p 53-62

[19] Barchielli A and Lupieri G 2000 J. Math. Phys. 41 7181-205

[20] Pero N 2000 Thesis Milan Univ., Phys. Dept.

[21] Ezekiel S and Wu F Y 1978 Multiphoton Processes edited by Eberly J H and Lambropoulos P (New York: Wiley) p 145-56

[22] Schuda F, Stroud C R Jr and Hercher M 1974 J. Phys. B: Atom. Molec. Phys. 7 L198-202

[23] Harting W, Rasmussen W, Schieder R and Walther H 1976 Z. Physik A 278 205-10

[24] Grove R E, Wu F Y and Ezekiel S 1977 Phys. Rev. A 15 227-33

[25] Cresser J D, Häger J, Leuchs G, Rateike M and Walther H 1982 Dissipative Systems in Quantum Optics, Topics in Current Physics Vol. 27 edited by Bonifacio R (Berlin: Springer) p 21-59

[26] Yuen H P and Shapiro J H 1978 IEEE Trans. Inf. Theory IT-24 657-68

[27] Messiah A 1970 Quantum Mechanics, Vol. II (Amsterdam: North-Holland)

[28] Shapiro J H, Yuen H P and Machado Mata J A 1979 IEEE Trans. Inf. Theory IT-25 179-92

[29] Barchielli A 1993 Classical and Quantum Systems - Foundations and Symmetries Proceedings of the II International Wigner Symposium edited by Doebner H D, Scherer W and Schroeck F Jr (Singapore: World Scientific) p 488-91 